\title{
BMJ Open Recognition of mental disorders: findings from a cross-sectional study among medical students in Singapore
}

\author{
Louisa Picco, ${ }^{1}$ Esmond Seow, ${ }^{1}$ Boon Yiang Chua, ${ }^{1}$ Rathi Mahendran, ${ }^{2,3}$ \\ Swapna Verma, ${ }^{3,4}$ Siow Ann Chong, ${ }^{1}$ Mythily Subramaniam ${ }^{1}$
}

To cite: Picco L, Seow E, Chua BY, et al. Recognition of mental disorders: findings from a cross-sectional study among medical students in Singapore. BMJ Open 2017;7:e019038. doi:10.1136/ bmjopen-2017-019038

- Prepublication history and additional material for this paper are available online. To view these files, please visit the journal online (http://dx.doi. org/10.1136/bmjopen-2017019038).

Received 7 August 2017 Revised 29 September 2017 Accepted 26 October 2017

CrossMark

${ }^{1}$ Research Division, Institute of Mental Health, Singapore ${ }^{2}$ Department of Psychological Medicine, Yong Loo Lin School of Medicine, National University of Singapore, Singapore

${ }^{3}$ Department of Clinical, Academic and Faculty Affairs, Duke-NUS Graduate Medical School, Singapore

${ }^{4}$ Department of General Psychiatry and Early Psychosis Intervention Programme, Institute of Mental Health, Singapore

Correspondence to Mythily Subramaniam; Mythily@imh.com.sg

\section{ABSTRACT}

Objectives To assess recognition of five mental

disorders (alcohol abuse, dementia, depression, obsessive-compulsive disorder (OCD) and schizophrenia) among a sample of medical students using a vignettebased approach. Socio-demographic predictors of correct recognition were also explored.

Design Cross-sectional online survey.

Participants Medical students studying in Singapore. Methods This was a cross-sectional online study among medical students $(n=502)$ who were randomly assigned one of the five vignettes. Students were instructed to read the vignette, then answer the open text question, 'What do you think the person in the vignette is suffering from?' Multiple logistic regression was performed to determine the predictors of correct recognition.

Results $81.7 \%$ could correctly recognise the condition described in the vignette. Depression was most well recognised $(93.0 \%)$, followed by alcohol abuse (89.0\%), OCD $(87.1 \%)$ and dementia $(79.2 \%)$, while only $60.0 \%$ of students correctly recognised schizophrenia. Females were significantly more likely to correctly recognise the disorders, while the odds of correct recognition were significantly higher among fourth-year and fifth-year students compared with first-year students. Compared with depression, dementia and schizophrenia were significantly more likely to be mislabelled.

Conclusion While overall correct recognition was high $(81.7 \%)$, this did vary by disorder, where schizophrenia $(60 \%)$ was the most poorly recognised condition. Given that primary care providers are often the first professional help-seeking source for people with mental health problems, medical students should be equipped with the skills and ability to recognise signs and symptoms of various mental illnesses.

\section{INTRODUCTION}

Mental illnesses cause tremendous human, social and economic burden worldwide, and this has been consistently substantiated in the extant literature. For example, WHO found that the prevalence of mental disorders ranged from $12 \%$ to $47 \%$ with most countries reporting a lifetime prevalence of at least one in four people. ${ }^{1}$ Recent estimates have also revealed that the global burden of

\section{Strengths and limitations of this study}

- A vignette-based approach was adopted to assess recognition relating to alcohol abuse, dementia, depression, obsessive-compulsive disorder and schizophrenia.

- This is the first study to explore recognition among a sample of Asian medical students across various psychiatric disorders.

- Multiple logistic regression allowed for predictors of correct recognition to be determined.

- The study has some limitations including the crosssectional design and lacks generalisability due to inclusion criteria.

mental illness accounts for $32.4 \%$ of years lived with disability (YLDs) and 13\% of disability-adjusted life-years (DALYs), placing mental illnesses as the largest global burden of disease in terms of YLDs, and equal to that of cardiovascular and circulatory diseases in terms of DALYs. ${ }^{2}$ Then there is the actual cost of mental illness; the Global Economic Burden of Non-communicable Diseases report showed mental disorders to be the largest cost driver, equating to $\$ 2.5$ trillion in global costs in 2010, where the costs for mental disorders were greater than the costs of diabetes, respiratory disorders and cancer combined. ${ }^{3}$

The impact of mental illness not only has a significant social and economic burden on society but the direct impact on people with mental illness is also extensive. A large body of evidence has consistently shown outcomes for people with mental illness are often much poorer ${ }^{4}$ in terms of mortality, morbidity $^{5}$ and access to appropriate services. ${ }^{6}$ Mental illness also impacts on the psychosocial facets of life such as education, employment and social relationships, ${ }^{7}$ often resulting in poorer quality of life, lower self-esteem and a sense of hopelessness. A myriad of factors are likely to contribute 
to these poorer psychosocial outcomes, of which two significant aspects include stigma and poor mental health literacy. ${ }^{89}$

Mental health literacy refers to 'knowledge and beliefs about mental disorders, which aid their recognition, management or prevention'. ${ }^{10}$ Equipping people with the skills and knowledge to identify the signs and symptoms of mental illness is imperative and has been linked to early help-seeking which can ultimately reduce the burden of disease associated with mental disorders. ${ }^{11}$ Despite this, it is not uncommon for people to be unable to recognise common signs and symptoms of mental disorders, and recognition can also vary considerably across mental illnesses. A recent national study in Singapore, which adopted a vignette-based approach to explore mental health literacy relating to five disorders, alcohol abuse, dementia, depression, obsessive-compulsive disorder (OCD) and schizophrenia, revealed that under half the Singapore population $(43.7 \%)$ could correctly recognise mental illnesses. The most well-recognised disorder was dementia $(66.3 \%)$, followed by alcohol abuse $(57.1 \%)$ and depression $(55.2 \%)$, while only $28.7 \%$ and $11.5 \%$ could correctly recognise OCD and schizophrenia, respectively. ${ }^{12}$

Far less is known about the mental health literacy of medical students in Singapore. While very few studies have explored mental health literacy solely among medical students, studies have investigated this concept among university and college students. Findings have revealed that in comparison medical students could better recognise mental illnesses ${ }^{13}$ or had better mental health literacy than students studying within other disciplines. ${ }^{14}{ }^{15}$ Despite this, medical students report feeling underprepared to manage mental health problems. ${ }^{16}$ Chur-Hansen et al, ${ }^{17}$ in their review of the medical education literature, revealed that most medical students receive limited and insufficient behavioural health education and training.

Given the severe shortage of specialist psychiatric care worldwide, primary care has been dubbed the de facto mental healthcare system. ${ }^{18}$ Primary care providers are therefore often the first point of contact for many people with mental illness. ${ }^{19}$ Despite this, mental health problems often go undiagnosed or undetected by primary care providers. ${ }^{2021}$ As part of their course curricula, it is imperative that adequate knowledge is imparted to medical students, who will be part of the future healthcare workforce, and hence need to be equipped with the skills and ability to recognise signs and symptoms of mental illness.

The current study aimed to assess recognition of five mental disorders (alcohol abuse, dementia, depression, OCD and schizophrenia) among a sample of medical students in Singapore. Socio-demographic predictors of correct recognition were also explored. These five disorders were selected based on various factors including their relatively high prevalence in the local population, the large treatment gap associated with them, ${ }^{22}{ }^{23}$ as well as the strong case for early detection and treatment of conditions such as psychosis which significantly impact outcomes. $^{24}$

\section{METHODS \\ Study participants}

Students from two medical schools in Singapore were informed of and invited to participate in the study via their institutional email. In all, 502 medical students were recruited during the period from August to September 2016. Limits were set across groups to ensure adequate representation across institutions and academic year levels. Additionally, medical students were required to be Singapore citizens or permanent residents and aged 16-35 years in order to be eligible to participate. The survey was administered via an online platform; all participants were required to provide informed consent, which was obtained when students read and indicated they were willing to partake in the study by clicking on the 'agree' link in the online consent form.

\section{Survey}

A structured questionnaire was used to gather socio-demographic information pertaining to the student's age, gender, ethnicity and academic year in addition to specific questions relating to their interest in psychiatry prior to starting medical school. To assess mental health literacy, a vignette-based approach was adopted, which modelled the Depression Literacy Questionnaire by Jorm $e t a l^{10}$ and that of a recent national mental health literacy study in Singapore. ${ }^{12}$ Students were randomly assigned one of five vignettes, which described a person with alcohol abuse, dementia, depression, OCD or schizophrenia. Vignettes were approximately 150 words in length and described classic and common symptoms of the five respective disorders. All vignettes were developed and revised in consultation with experienced research psychiatrists, specialising in each of the five disorders, and then further vetted by a panel of senior clinical psychiatrists to ensure they reflected Diagnostic and Statistical Manual of Mental Disorders, 4th edition and International Statistical Classification of Diseases and Related Health Problems, 10th revision diagnostic criteria for the five disorders. These vignettes were also cognitively tested prior to their use, where trained interviewers systematically probed respondents on what they thought the vignette was about, what came to their mind when they heard a particular phrase or term and whether there were any words they did not understand and any words or expression that they found offensive or unacceptable. Where alternative words or expressions exist for certain terms, the respondent was asked which of the alternatives conforms better to their usual language. Changes were made to the language of the vignettes where necessary after summarising the results of all the cognitive interviews. The five vignettes pertaining to this study have been included in online supplementary file 1 . 
After reading the assigned vignette, students were asked a series of questions relating to the person in the vignette. They were asked an open text question: 'What do you think the person in the vignette is suffering from?' which was used to ascertain whether students could accurately recognise or name the mental illness being described. In addition, students were also asked to indicate if anyone in their family or close circle of friends had ever had problems similar to the person in the vignette and if they had any experience in dealing with a person with problems similar to those described in the vignette. The current study was a part of a larger study that explored mental health literacy and factors associated with choosing psychiatry practice as a career and the entire survey took on average $30-40 \mathrm{~min}$ to complete.

\section{Coding}

Two members of the research team (LP and ES) independently coded the open text responses in relation to correct recognition. Responses were coded as 'Correct recognition' if the respondent was able to accurately name the specific condition. The two coders then compared responses to ensure consistency, and in the case of an ambiguous response, the two coders (LP and ES) would come to a consensus on how the response should be coded. First, responses were coded as either being 'correct' or 'incorrect'. For those responses that were incorrect or mislabelled, these were further classified as (i) disorder-specific symptoms, (ii) other mental disorder (anxiety), (iii) other mental disorder (depression), (iv) other mental disorder (miscellaneous), (v) mental illness (not specified), (vi) psychological stress, (vii) not an illness and (viii) don't know/irrelevant response and were similar to codes used in an earlier national mental health literacy study that used the same vignettes. ${ }^{12}$ Responses pertaining to 'disorder-specific symptoms' included short-term memory/memory loss or mild cognitive impairment for dementia, germophobia for OCD and hallucinations, delusions or paranoia for schizophrenia. 'Not an illness' refers to responses such as loneliness or lack of social interaction.

\section{Statistical analysis}

Statistical analyses were performed using IBM SPSS V.23.0. Descriptive statistics were tabulated for the overall sample, with frequency and percentage calculated for all categorical variables. Given the exploratory nature of the current study, multiple logistic regression using the enter method was performed to determine the predictors of correct recognition as this would take into account the effects of all predictors and select the stronger covariates. This generated ORs and 95\% CIs for the relationship between correct recognition (dependent variable) and various predictors including age group, gender, ethnicity, academic year, vignette type, interest in psychiatry prior to medical school, experience dealing with someone who had similar mental health problems and family or friends who have similar

\begin{tabular}{|c|c|c|}
\hline & $\mathbf{n}$ & $\%$ \\
\hline \multicolumn{3}{|c|}{ Age group (years) } \\
\hline$<21$ & 154 & 30.7 \\
\hline$\geq 21$ & 348 & 69.3 \\
\hline \multicolumn{3}{|l|}{ Gender } \\
\hline Male & 207 & 41.2 \\
\hline Female & 295 & 58.8 \\
\hline \multicolumn{3}{|l|}{ Ethnicity } \\
\hline Chinese & 467 & 93.0 \\
\hline Non-Chinese & 35 & 7.0 \\
\hline \multicolumn{3}{|l|}{ Academic year } \\
\hline First & 132 & 26.3 \\
\hline Second & 116 & 23.1 \\
\hline Third & 71 & 14.1 \\
\hline Fourth & 87 & 17.3 \\
\hline Fifth & 96 & 19.1 \\
\hline \multicolumn{3}{|c|}{ Interest in psychiatry prior to medical school } \\
\hline Yes & 20 & 4.0 \\
\hline No & 482 & 96.0 \\
\hline \multicolumn{3}{|c|}{$\begin{array}{l}\text { Has experience dealing with someone having problems } \\
\text { similar to ' } X \text { ' }\end{array}$} \\
\hline Yes & 127 & 25.3 \\
\hline No & 375 & 74.7 \\
\hline \multicolumn{3}{|c|}{ Has friends and family with problems similar to ' $\mathrm{X}$ ' } \\
\hline Yes & 164 & 32.7 \\
\hline No & 338 & 67.3 \\
\hline
\end{tabular}

problems to those in the vignette. Statistical significance was set at $\mathrm{P}<0.05$ level.

\section{RESULTS}

The sample characteristics of the medical students are displayed in table 1 . The majority of the sample were $>21$ years of age $(69.3 \%)$, female $(58.8 \%)$ and Chinese (93.0\%). $25.3 \%$ of students had experience in dealing with problems similar to those described in the vignette, and $32.7 \%$ reported they had friends or family with similar problems.

Table 2 shows the percentage of respondents endorsing each category in relation to recognition of the vignettes. In total, $81.7 \%$ could correctly recognise the condition described in the vignette, where depression was the most well recognised $(93.0 \%)$, followed by alcohol abuse $(89.0 \%)$, OCD $(87.1 \%)$ and dementia $(79.2 \%)$, while only $60.0 \%$ of students correctly recognised schizophrenia. In relation to schizophrenia, students commonly used terms to describe the symptoms of the disorders such as hallucinations or delusions $(12.0 \%)$ or mislabelled this as another mental illness such as delusional disorder or autism $(10 \%)$. 
Table 2 Percentage of medical students mentioning each category to describe the problem in the vignette

\begin{tabular}{|c|c|c|c|c|c|c|}
\hline & $\begin{array}{l}\text { Total } \\
(n=502)\end{array}$ & $\begin{array}{l}\text { Alcohol } \\
\text { abuse } \\
(n=100)\end{array}$ & $\begin{array}{l}\text { Dementia } \\
(n=101)\end{array}$ & $\begin{array}{l}\text { Depression } \\
(n=100)\end{array}$ & $\begin{array}{l}\text { Obsessive- } \\
\text { compulsive } \\
\text { disorder } \\
(\mathrm{n}=101)\end{array}$ & $\begin{array}{l}\text { Schizophrenia } \\
(n=100)\end{array}$ \\
\hline \multicolumn{7}{|l|}{ Recognition } \\
\hline Correct recognition & 81.7 & 89.0 & 79.2 & 93.0 & 87.1 & 60.0 \\
\hline Disorder-specific symptoms & 5.6 & - & 9.9 & 1.0 & 5.0 & 12.0 \\
\hline Other mental disorder-any anxiety disorder & 2.2 & 1.0 & 1.0 & - & 3.0 & 6.0 \\
\hline Other mental disorder-depression & 3.8 & 8.0 & 5.9 & - & 1.0 & 4.0 \\
\hline Other mental disorder-miscellaneous & 3.0 & 1.0 & 1.0 & 1.0 & 2.0 & 10.0 \\
\hline Mental illness & 1.4 & - & 1.0 & 2.0 & 1.0 & 3.0 \\
\hline Psychosocial stress & 1.0 & - & 1.0 & 3.0 & 1.0 & - \\
\hline Not an illness & 1.0 & 1.0 & 1.0 & - & - & 3.0 \\
\hline Not sure/irrelevant response & 0.4 & - & - & - & - & 2.0 \\
\hline
\end{tabular}

Predictors of correct recognition of mental disorders are shown in table 3. Multiple logistic regression analyses revealed that females $(\mathrm{P}=0.013)$ were significantly more likely to correctly recognise the disorder being described in the vignette, while the odds of correct recognition were significantly higher among fourth-year $(\mathrm{P}=0.019)$ and fifth-year students $(\mathrm{P}<0.001)$ compared with first-year students. Differences across vignettes were also observed. When compared with depression, the dementia $(\mathrm{P}=0.009)$ and schizophrenia vignettes $(\mathrm{P}<0.001)$ were significantly more likely to be mislabelled.

\section{DISCUSSION}

This mental health literacy study among medical students has explored recognition rates for common mental disorders, namely alcohol abuse, dementia, depression, OCD and schizophrenia, using a vignette-based approach. The study also sought to identify socio-demographic predictors of correct recognition. A similar protocol was used in earlier mental health literacy studies in Singapore, allowing for comparisons in recognition to be made across samples. Findings from the current study revealed that correct recognition of mental disorders overall was quite high $(81.7 \%)$. In comparison to other local studies, recognition was considerably higher than that of the general Singapore population $(43.7 \%)^{12}$ and slightly higher than that of a nursing student sample $(70.4 \%) .{ }^{25}$

Correct recognition did, however, vary across disorders, with the most well-recognised disorder being depression $(93.0 \%)$, followed by alcohol abuse, $(89.0 \%)$, OCD $(87.1 \%)$ and dementia $(79.2 \%)$ while the most poorly recognised condition was schizophrenia $(60.0 \%)$. Regression analysis further substantiated this where compared with depression, medical students were significantly less likely to correctly recognise dementia $(\mathrm{P}=0.009)$ and schizophrenia $(\mathrm{P}<0.001)$. The latter corroborates findings of other local mental health literacy studies among the general population ${ }^{12}$ and nursing students $^{25}$ in
Singapore, which also found recognition was poorest for schizophrenia in comparison to the other four disorders. Mental health literacy studies elsewhere have also found schizophrenia to be more poorly recognised compared with depression. ${ }^{26} 27$

Schizophrenia recognition is consistently poor across different population subgroups in Singapore, and this, coupled with its severity and chronicity, impacts patients, their families and the wider community. In addition, 20\% of medical students also incorrectly identified this as another mental illness (eg, depression, anxiety, delusional disorder). As expected, further analysis revealed that both incorrect recognition and mislabelling schizophrenia for another mental illness were most common in first-year students, however it still occurred more frequently in fourth-year and fifth-year students, compared with the other vignettes, and therefore course curricula pertaining to psychiatry may benefit from focusing on specific symptomology of schizophrenia, given recognition was poorest for this condition.

With regards to dementia recognition among medical students, this was higher than that of the general population $(79.2 \%$ vs $66.3 \%)$-where dementia was the most well-recognised disorder ${ }^{12}$-and similar to that of nursing students (77\%). ${ }^{25}$ However, compared with the other disorders, it was the second most poorly recognised disorder after schizophrenia. One in 10 students described disorder-specific symptoms such as short-term memory/memory loss or mild cognitive impairment and $5.9 \%$ incorrectly recognised it as depression. Similarly, $8 \%$ and $4 \%$ of students also mislabelled alcohol abuse and schizophrenia as depression, respectively. So while depression was very well recognised among medical students, it was also 'overgeneralised' and used to incorrectly label all four of the other disorders, a finding which is consistent with local and international studies. 122526 Although it is important that medical students can identify the person as having a mental illness, it is important 
Table 3 Predictors of correct recognition of mental disorders $(n=502)$

\begin{tabular}{lcccc}
\hline & OR & Lower & Upper & P value \\
\hline $\begin{array}{l}\text { Age group (years) } \\
<21\end{array}$ & 1.02 & 0.54 & 1.94 & 0.949 \\
$\geq 21$ & Ref. & - & - & - \\
Gender & & & & \\
\hline Female & 1.91 & 1.15 & 3.18 & 0.013 \\
Male & Ref. & - & - & - \\
\hline Ethnicity & & & & \\
Chinese & Ref. & - & - & - \\
\hline Non-Chinese & 0.55 & 0.23 & 1.34 & 0.188 \\
\hline Academic year & & & & \\
\hline First & Ref. & - & - & - \\
\hline Second & 1.15 & 0.61 & 2.16 & 0.672 \\
\hline Third & 1.70 & 0.72 & 4.03 & 0.228 \\
\hline Fourth & 2.97 & 1.20 & 7.36 & 0.019 \\
\hline Fifth & 6.52 & 2.24 & 19.03 & $<0.001$ \\
\hline
\end{tabular}

Interest in psychiatry prior to medical school

\begin{tabular}{lcccc} 
Yes & 2.28 & 0.46 & 11.39 & 0.316 \\
No & Ref. & - & - & - \\
Vignette type & & & & \\
Depression & Ref. & - & - & - \\
Dementia & 0.28 & 0.11 & 0.73 & 0.009 \\
Alcohol abuse & 0.58 & 0.20 & 1.64 & 0.301 \\
$\begin{array}{l}\text { Obsessive- } \\
\text { compulsive }\end{array}$ & 0.48 & 0.17 & 1.36 & 0.167 \\
disorder & & & & \\
$\begin{array}{l}\text { Schizophrenia } \\
\text { Experience dealing }\end{array}$ & 0.09 & 0.04 & 0.23 & $<0.001$ \\
No someone similar to 'X' & \\
Yes & Ref. & - & - & - \\
Has friends and family with problems similar to 'X' & \\
No & Ref. & - & - & - \\
Yes & 0.90 & 0.46 & 1.78 & 0.764 \\
\hline
\end{tabular}

they can differentiate the symptoms of depression from that of other mental illnesses.

Correct recognition of both alcohol abuse $(89 \%)$ and OCD $(87.1 \%)$ was high among medical students. When comparing correct recognition rates to that of the general Singapore population and nursing students, the greatest differences were also observed for these disorders; $87.1 \%$ of medical students correctly recognised OCD, versus just $28.7 \%$ of the general population, ${ }^{12}$ while $89 \%$ correctly labelled alcohol abuse compared with $58 \%$ of nursing students. ${ }^{25}$ Correct recognition for OCD among the general population was quite poor, which is likely to be a result of less emphasis being placed on this mental illness in the local media, compared with conditions such as depression or dementia, and consequently, the general population are less familiar with the term 'obsessive-compulsive disorder' or 'OCD'. In addition, $15 \%$ of Singaporeans did not think this was a problem, ${ }^{12}$ which somewhat normalises the symptoms and may further explain the contrasting recognition rates. Conversely, alcohol abuse was the second most well-recognised condition among medical students and the general population, yet was the second most poorly recognised condition among nursing students after schizophrenia. It is possible that medical students may have the ability to more objectively assess recognition based on the symptoms described in the vignette or their course curriculum may provide them the skills to better identify the symptoms of alcohol abuse compared with nursing students.

The current study identified a number of socio-demographic predictors of correct recognition, including gender and academic year level, as well as experience in dealing with similar problems to those described in the vignette. Females were nearly two times more likely to correctly recognise the disorder described in the vignette compared with their male counterparts. Examination of gender differences in mental health literacy have consistently found that females are better able to recognise the signs and symptoms of mental illnesses compared with males, in university student populations, ${ }^{15} 27$ adolescents and young adults, ${ }^{28}$ as well as adult populations. ${ }^{12}$ These findings have been attributed to females often having greater awareness of symptoms, whereas males are less aware of health problems. More specifically, females have been shown to be more intuitive than males in relation to emotional understanding and therefore may be more willing to use psychological labels than their male counterparts, ${ }^{29}$ while males are generally less likely to value assistance from health professionals. ${ }^{28}$ Accordingly, consideration should be given to how course content is communicated and whether there is a need for different types of information and educational strategies to better target these gender differences. ${ }^{28}$

As expected, fourth-year and fifth-year medical students were significantly more likely to correctly recognise the disorder described in the vignette compared with students in their first year of medicine, a finding which concurs with previous research. ${ }^{13}$ Medical school curricula for first-year students only include very limited and preliminary information relating to psychiatry, where the majority of psychiatric teaching clerkship and placements occurs in the latter years of the course. Therefore, it is not surprising that the knowledge and ability to correctly recognise signs and symptoms of various disorders among first-year students is poorer than students in their final years of medicine. This finding also lends itself to the importance and impact of undertaking clinical psychiatry placements and how the experience coupled with psychiatry education in these final years of a medicine course can contribute to significantly better recognition in fourth-year and fifth-year students.

Previous experience in dealing with problems similar to those described in the vignette was also associated with 
better recognition, and although this finding was not significant, it was approaching significance $(\mathrm{P}=0.056)$. Students were not explicitly asked about the type or duration of their experience in dealing with people with mental illness; however, this could be in the form of volunteering or work experience, helping family or friends or they may have gained exposure through practical placements as part of their course. The literature has also consistently reported that experience or exposure in dealing with someone who has a mental illness also results in improved mental health literacy. ${ }^{15}$ Furthermore, studies have also found that history of personal contact with people with mental health issues is also associated with reduced stigma and improved attitudes towards people with mental illness. ${ }^{30} 31$

When interpreting these findings, it is also important to consider the socio-demographic characteristics of the person in the vignette and how these may impact and influence correct recognition. More specifically, factors such as gender, age and race/ethnicity ${ }^{32}{ }^{33}$ of the person depicted in the vignette have been found to influence recognition. Similarly, a vignette-based study found that patient characteristics and factors play a role in the decision to provide self-management support among primary care physicians and nurses. ${ }^{34}$ In the current study, the gender for all vignettes was male, ethnicity was not stipulated, and nor was there any specific reference to socioeconomic indicators; future studies could benefit from incorporating such information in the vignette to see if such characteristics influence recognition.

Some limitations must be acknowledged in view of the current findings. The vignettes used describe classic symptoms of each of the five disorders but may not describe all symptoms or reflect real-life cases. As the current sample are future medical professionals, further exploration of student's ability to recognise more complicated cases such as those with comorbidities, and those with prodromal or uncommon symptoms is recommended. The sample was also restricted to Singapore citizens and permanent residents, and therefore, the findings may not be generalisable to international medical students studying in Singapore. Finally, correct recognition was based on students correctly identifying one of five randomly assigned vignettes, describing someone with alcohol abuse, dementia, depression, OCD or schizophrenia. Future studies could assign multiple or all vignettes to the same student in order to gain a greater and more in-depth understanding of recognition rates across disorders and how these may differ.

These limitations notwithstanding, the current study recruited a reasonable sample of medical students, whereby correct recognition was assessed across common mental illnesses. It adopted a similar protocol to that of a local national mental health literacy study ${ }^{12}$ allowing for comparisons to be made between the general population as well as among a sample of nursing students. ${ }^{25}$ While overall recognition was high $(81.7 \%)$, disorders such as schizophrenia were more poorly recognised
$(60 \%)$, highlighting the need for greater emphasis and increased awareness on such aspects given the severity of this mental illness. Furthermore, predictors of correct recognition were also identified such as being female and having previous experience in dealing with mental health problems. Accordingly, gender-specific interventions should be considered while providing exposure or contact with people with mental illness would be beneficial to not only improve recognition and overall mental health literacy, but also in reducing stigma and improving attitudes towards people with mental illness. ${ }^{31}$

The implications of medical student's mental health literacy are significant. Given that these students are the next generation of doctors, it is imperative that they are equipped with the skills and ability to recognise signs and symptoms of mental illness, especially given primary care providers are often the first professional help-seeking source for people with mental health problems. ${ }^{19}{ }_{22}$ Ongoing consideration should also be given to ensure medical school psychiatry education and curricula are routinely reviewed and updated in order to assist in the preparation of qualifying doctors to successfully recognise and manage common mental disorders ${ }^{35}$ on entering the workforce. Furthermore, given that students in their final years of their degree (vs first year) were significantly better able to recognise mental disorders, this substantiates the importance of psychiatric clinical placements in terms of knowledge and recognition of such conditions.

As appropriate and timely help-seeking is associated with improved long-term outcomes for people with mental illness, ${ }^{36}$ it is fundamental that the future medical workforce gain knowledge and psychiatric exposure as part of their medical course, with the long-term goal to improve outcomes for people with mental illness and ultimately the wider community at large. Furthermore, as recognition of schizophrenia was poorest and often mislabelled as other common mental illnesses, increased efforts are needed to better educate medical students, about specific signs and symptoms of individual mental disorders, so that they can differentiate between these in future.

Contributors LP was responsible for writing the manuscript. ES was responsible for the study design, data analysis and provided inputs into the manuscript. BYC was responsible for the study design and provided inputs into the manuscript. RM and SV provided inputs into data interpretation and edits to the manuscript. SAC and MS supervised the overall study and provided intellectual inputs on the manuscript.

Funding This research was supported by the Singapore Ministry of Health's National Medical Research Council under the Centre Grant Programme (grant no.: 10.13039/501100001349 NMRC/CG/004/2013).

Competing interests None declared.

Patient consent Obtained.

Ethics approval Ethical approval was granted from the relevant institutional review board (National Healthcare Group, Domain Specific Review Board).

Provenance and peer review Not commissioned; externally peer reviewed.

Data sharing statement Readers who wish to gain access to the data can write to the senior author MS at mythily@imh.com.sg. 
Open Access This is an Open Access article distributed in accordance with the Creative Commons Attribution Non Commercial (CC BY-NC 4.0) license, which permits others to distribute, remix, adapt, build upon this work non-commercially, and license their derivative works on different terms, provided the original work is properly cited and the use is non-commercial. See: http://creativecommons.org/ licenses/by-nc/4.0/

(c) Article author(s) (or their employer(s) unless otherwise stated in the text of the article) 2017. All rights reserved. No commercial use is permitted unless otherwise expressly granted.

\section{REFERENCES}

1. Kessler RC, Angermeyer M, Anthony JC, et al. Lifetime prevalence and age-of-onset distributions of mental disorders in the World Health Organization's World Mental Health Survey Initiative. World Psychiatry 2007;6:168-76.

2. Vigo D, Thornicroft G, Atun R. Estimating the true global burden of mental illness. Lancet Psychiatry 2016;3:171-8.

3. Bloom DE, Cafiero ET, Jané-Llopis E, et al. The Global Economic Burden of Noncommunicable Diseases. Geneva: World Economic Forum, 2011

4. Thornicroft G. Physical health disparities and mental illness: the scandal of premature mortality. Br J Psychiatry 2011;199:441-2.

5. Prince M, Patel V, Saxena S, et al. No health without mental health. The Lancet 2007;370:859-77.

6. Demyttenaere K, Bruffaerts R, Posada-Villa J, et al. Prevalence, severity, and unmet need for treatment of mental disorders in the World Health Organization World Mental Health Surveys. JAMA 2004;291:2581-90.

7. The Organisation for Economic Co-operation and Development (OECD). Sick on the Job? Myths and Realities about Mental Health and Work. OECD Publishing, 2012.

8. Jorm AF. Mental health literacy. Public knowledge and beliefs about mental disorders. Br J Psychiatry 2000;177:396-401.

9. Hatzenbuehler ML, Phelan JC, Link BG. Stigma as a fundamental cause of population health inequalities. Am J Public Health 2013;103:813-21.

10. Jorm AF, Korten AE, Jacomb PA, et al. 'Mental health literacy': a survey of the public's ability to recognise mental disorders and their beliefs about the effectiveness of treatment. Med J Aust 1997;166:182-6.

11. Wang PS, Berglund $\mathrm{P}$, Olfson $\mathrm{M}$, et al. Failure and delay in initial treatment contact after first onset of mental disorders in the National Comorbidity Survey Replication. Arch Gen Psychiatry 2005;62:603-13.

12. Chong SA, Abdin E, Picco L, et al. Mental Health Literacy among a multiracial population in South East Asia. BMC Psychiatry 2016;16:121.

13. Amarasuriya SD, Jorm AF, Reavley NJ. Quantifying and predicting depression literacy of undergraduates: a cross sectional study in Sri Lanka. BMC Psychiatry 2015;15:269.

14. Furnham A, Cook R, Martin N, et al. Mental health literacy among university students. J Public Ment Health 2011;10:198-210.

15. Lauber C, Ajdacic-Gross V, Fritschi N, et al. Mental health literacy in an educational elite - an online survey among university students. BMC Public Health 2005;5:44.

16. Sahhar D, O'Connor D. How well do Australian medical schools prepare general practitioners to care for patients with mental disorders? Australas Psychiatry 2004;12:26-30.
17. Chur-Hansen A, Carr JE, Bundy C, et al. An international perspective on behavioral science education in medical schools. J Clin Psychol Med Settings 2008;15:45-53.

18. Kessler R, Stafford D. Collaborative Medicine Case Studies. SpringerVerlag New York, 2008.

19. Bristow K, Edwards S, Funnel E, et al. Help seeking and access to primary care for people from "hard-to-reach" groups with common mental health problems. Int J Family Med 2011;2011:1-10.

20. Smith DJ, Griffiths E, Kelly M, et al. Unrecognised bipolar disorder in primary care patients with depression. Br J Psychiatry 2011;199:49-56.

21. Bor JS, elderly Athe. many mental illnesses go undiagnosed. Health Aff 2015;34:727-31.

22. Chong SA, Abdin E, Vaingankar JA, et al. A population-based survey of mental disorders in Singapore. Ann Acad Med Singapore 2012;41:49-66.

23. Subramaniam M, Chong SA, Vaingankar JA, et al. Prevalence of dementia in people aged 60 years and above: results from the WiSE Study. J Alzheimers Dis 2015;45:1127-38.

24. Verma S, Subramaniam M, Abdin E, et al. Symptomatic and functional remission in patients with first-episode psychosis. Acta Psychiatr Scand 2012;126:282-9.

25. Seow LSE, Chua BY, Xie H, et al. Correct recognition and continuum belief of mental disorders in a nursing student population. BMC Psychiatry 2017;17:289.

26. Reavley NJ, Jorm AF. Recognition of mental disorders and beliefs about treatment and outcome: findings from an Australian national survey of mental health literacy and stigma. Aust N Z J Psychiatry 2011;45:947-56.

27. Gorczynski P, Sims-schouten W, Hill D, et al. Examining mental health literacy, help seeking behaviours, and mental health outcomes in UK university students. The Journal of Mental Health Training, Education and Practice 2017;12:111-20.

28. Cotton SM, Wright A, Harris MG, et al. Influence of gender on mental health literacy in young Australians. Aust N Z J Psychiatry 2006;40:790-6.

29. Burns JR, Rapee RM. Adolescent mental health literacy: young people's knowledge of depression and help seeking. J Adolesc 2006;29:225-39.

30. Subramaniam M, Abdin E, Picco L, et al. Stigma towards people with mental disorders and its components - a perspective from multiethnic Singapore. Epidemiol Psychiatr Sci 2017;26:1-12.

31. Evans-Lacko S, London J, Japhet S, et al. Mass social contact interventions and their effect on mental health related stigma and intended discrimination. BMC Public Health 2012;12:489.

32. Hadjimina E, Furnham A. Influence of age and gender on mental health literacy of anxiety disorders. Psychiatry Res 2017;251:8-13.

33. Morley CP. The effects of patient characteristics on ADHA diagnosis and treatment: a factorial study of family physicians. BMC Family Practice 2010:11:11.

34. Bos-Touwen ID, Trappenburg JC, van der Wulp I, et al. Patient factors that influence clinicians' decision making in selfmanagement support: A clinical vignette study. PLoS One 2017;12:e0171251.

35. Thomas S, Pai N, Dawes K, et al. Updating medical school psychiatry curricula to meet projected mental health needs. Australas Psychiatry 2013;21:578-82

36. McGorry PD, Purcell R, Hickie IB, et al. Investing in youth mental health is a best buy. Med J Aust 2007;187:S5-7. 